\title{
Blunted Corticosterone Response to Acute Predator Stress Results in Long-Term Spatial Memory Impairment
}

\author{
Phillip R. Zoladz ${ }^{1 *}$, Hanna Burke ${ }^{1}$, Cristina Robinson ${ }^{1}$, Sarah Woelke ${ }^{1}$, Bethany Wentz ${ }^{1}$, Julia \\ Pisansky ${ }^{1}$, Jerel McKay ${ }^{1}$, Kyle Dexter ${ }^{1}$ and Jeffery N. Talbot ${ }^{2}$ \\ ${ }^{1}$ Department of Psychology, Sociology, \& Criminal Justice, Ohio Northern University, Ada, Ohio, United States \\ ${ }^{2}$ College of Pharmacy and Program for Novel Therapeutics in Neurological and Psychiatric Disorders, Roseman University of Health Sciences, \\ Henderson, Nevada
}

Received: October 29, 2013; Accepted: J anuary 04, 2014; Published: J anuary 06, 2014

*Corresponding author: Phillip R Zoladz, Department of Psychology, Sociology, \& Criminal Justice, Ohio Northern University, 525 S. Main St., Ada, Ohio, USA, Tel: 419-772-2142; Fax: 419-772-2746; Email: p-zoladz@onu.edu

\begin{abstract}
Clinical research suggests that a blunted corticosteroid response to trauma may be associated with increased risk of developing post-traumatic stress disorder (PTSD). Therefore, we examined the influence of a blunted corticosterone response to stress on the development of PTSD-like behaviors, such as increased anxiety and impaired learning and memory, in rats. Male Sprague-Dawley rats were injected with metyrapone, an inhibitor of corticosterone synthesis, or vehicle prior to being exposed to an adult female cat for one hour. One week later, the rats were tested for anxiety-like behaviors (e.g., reduced open arm exploration, decreased locomotor activity) on an elevated plus maze and for spatial learning and memory in the radial-arm water maze. Analyses of post-stress serum samples verified that metyrapone blocked the stressinduced increase of corticosterone levels. Behaviorally, the blunted corticosterone response to stress had no effect on the development of anxiety-like behaviors. On the other hand, we found that metyrapone administration prior to stress significantly impaired long-term spatial memory. These findings suggest that reduced corticosteroid levels could interact with stress to produce deleterious effects on cognitive performance. Moreover, because anxiety-like behaviors were not intensified by the blunted corticosteroid response to stress, our findings also suggest that specific physiological responses to an acute trauma may intensify some, but not all, PTSD-like symptoms.
\end{abstract} Stress

Keywords: Anxiety; Corticosterone; Memory; PTSD; Rat model;

\section{Introduction}

Individuals who are exposed to life-threatening trauma are at significant risk of developing post-traumatic stress disorder (PTSD), which is characterized by intrusive memories, persistent anxiety, an exaggerated startle response and cognitive impairments [1-6]. Importantly, not every individual who is exposed to trauma develops PTSD [7], suggesting that those who do develop PTSD may retain some sort of predisposing genetic factors for PTSD or may respond to the traumatic event in a way that makes them more likely to develop the disorder. Indeed, research has suggested that an individual's hypothalamus- pituitary-adrenal (HPA) axis response to a traumatic event could affect his or her likelihood of developing PTSD [8-10]. In these studies, researchers showed that individuals who developed PTSD exhibited significantly lower levels of cortisol after the traumatic event than those individuals who did not develop PTSD. Preclinical work has provided similar results by examining the development of PTSD-like behaviors in different strains of rats. For instance, Cohen and colleagues [11] reported that Lewis rats, which exhibit a significantly blunted corticosterone response to stress, are more prone to developing PTSD-like behaviors following an acute stressor than Fischer or SpragueDawley rats. However, the studies conducted on this topic thus far have not been able to determine whether it is the HPA axis response to an acute stressor that affects PTSD / PTSD-like behavior development or if reduced HPA axis activity is merely a baseline characteristic of the people / rats that are more likely to develop PTSD / PTSD-like behaviors.

Only one study of which we are aware has utilized an experimental inhibition of a rat's HPA axis response to stress in order to assess whether or not it would facilitate the development of PTSD-like behaviors. In that study, the investigators found that the combination of a blunted corticosterone and parasympathetic nervous system response to stress resulted in significantly greater anxiety on an elevated plus maze three weeks following a single stress exposure [12]. However, these results were observed when comparing the experimental groups to their respective controls only, and the effects of the manipulations on cognitive performance were not examined. This latter point is especially important because PTSD can be considered a disorder of memory, as individuals suffering from the disorder show marked impairments of learning and memory, a phenomenon that may be a result of intrusive memories transiently interfering with these individuals' ability to process new information [1317]. Thus, the purpose of the present study was to determine whether or not a blunted HPA axis response to acute predator 
stress would facilitate the development of PTSD-like symptoms, such as enhanced anxiety and impaired learning and memory, in rats. We predicted that stressed rats treated with a corticosteroid inhibitor would exhibit greater levels of anxiety and greater impairments of learning and memory than stressed rats treated with vehicle one week following stress exposure.

\section{Materials and Methods}

\section{Animals}

Male Sprague-Dawley rats $(\sim 150 \mathrm{~g})$ that were bred at Ohio Northern University were housed on a $12 \mathrm{~h} / 12 \mathrm{~h}$ light/dark schedule (lights on at 0700 hours) in Plexiglas cages ( 2 per cage) with free access to food and water. The Institutional Animal Care and Use Committee at Ohio Northern University approved all experimental procedures.

\section{Pharmacological agents}

Rats were injected intraperitoneally with metyrapone (150 $\mathrm{mg} / \mathrm{kg} ; \mathrm{N}=22)$ or vehicle $(30 \%$ polyethylene glycol solution; $\mathrm{N}$ $=21$ ) at a volume of $1 \mathrm{ml} / \mathrm{kg}$. Metyrapone is a cytochrome $\mathrm{P}_{450}$ inhibitor that prevents the conversion of deoxycorticosterone to corticosterone in the adrenal cortex [18].

\section{Stress procedure}

Thirty minutes following the injection, the rats were assigned to stress (metyrapone $\mathrm{N}=11$; vehicle $\mathrm{N}=10$ ) or no stress (metyrapone $\mathrm{N}=11$; vehicle $\mathrm{N}=11$ ) conditions. Rats in the stress condition were placed in a perforated wedge-shaped Plexiglas enclosure (Braintree Scientific; Braintree, MA; 20 × 20 × $8 \mathrm{~cm}$ ), which was placed in a small room $(2.41 \times 1.5 \times 2.26 \mathrm{~m})$ with an adult female cat for $1 \mathrm{hr}$ [19-22]. Rats in the no stress condition remained in their home cages for the same time frame.

\section{Blood sampling}

Following the stress / no stress manipulation, the saphenous vein of each rat was punctured with a sterile, 27-gauge syringe needle. A $0.2 \mathrm{cc}$ sample of blood was collected from each rat within $2 \mathrm{~min}$. The serum was subsequently assayed for corticosterone with an Enzyme ImmunoAssay kit from Enzo Life Sciences, Inc. (cat\# 901-097; Ann Arbor, MI). All samples were diluted 1:50 and assayed per kit instructions.

\section{Behavioral testing}

Elevated plus maze: One week following the stress / no stress manipulation, the rats were placed on an elevated plus maze (EPM) for $5 \mathrm{~min}$. The EPM is a routine assessment of anxiety [23] and consists of two open arms $(11 \times 50 \mathrm{~cm})$ and two closed $\operatorname{arms}(11 \times 50 \mathrm{~cm})$ that intersect each other to form the shape of a plus sign. Rat behavior was videotaped by a JVC hard disk camera hanging above the EPM and scored offline by investigators who were blind to the experimental conditions of the animals. The investigators assessed the following measures: time spent in the open and closed arms, number of entries into the open and closed arms, number of rearing episodes and number of open arm head dips. Anxiety-like behavior is often evidenced by a reduction of open arm exploration (time spent in the arms and entries into the arms), as well as a reduction of general behavioral activity (e.g., rearing) [24-25]. Open arm head dips, which are reflective of risk assessment behavior, are often reduced in stressed animals and can be another indicator of anxiety-like behavior. Closed arm time and entries are an indication of general locomotor activity, and in some cases, can be suppressed by stress as well.

Radial-Arm Water Maze: One day after EPM testing, rat spatial learning and memory was assessed in the radial-arm water maze (RAWM), a hippocampus-dependent task [19,20,22,2628]. The RAWM consisted of a black, galvanized round tank (168 cm diameter, $56 \mathrm{~cm}$ height, $43 \mathrm{~cm}$ depth) filled with water $\left(22^{\circ} \mathrm{C}\right)$. Six V-shaped stainless steel inserts $(54 \mathrm{~cm}$ height, $56 \mathrm{~cm}$ length; $60-70^{\circ}$ angle) were placed in the tank to produce six arms radiating from an open central area. A black, plastic platform $(12$ $\mathrm{cm}$ diameter) was placed $1 \mathrm{~cm}$ below the surface of the water at the end of one arm (referred to as the "goal arm"). The goal arm was different across rats to prevent the build-up of odor cues in any one arm. At the start of each trial, rats were released into one arm (referred to as the "start arm") facing the center of the maze and given $1 \mathrm{~min}$ to locate the platform. The start arm was pseudorandomly changed after each trial so that it was never the same for two consecutive trials. Several extra-maze cues (e.g., posters) were on the walls of the room to facilitate rat navigation in the maze. Once on the platform, rats were left undisturbed for 15 sec. If, during any trial, a rat did not locate the hidden platform within $1 \mathrm{~min}$, it was gently guided to the platform by the experimenter. Spatial learning and memory was measured by counting the number of arm entry errors that rats made on each trial. An arm entry was operationally defined as a rat passing at least halfway down the arm; an arm entry error consisted of a rat entering on the of non-goal arms or, very rarely, a rat entering and exiting the goal arm without climbing onto the platform. The rats were given 12 acquisition trials to learn the location of the hidden platform, followed $60 \mathrm{~min}$ later by a single shortterm memory trial. On the following day, the rats were given one more trial in the RAWM to assess their long-term memory for the platform location.

\section{Statistical analyses}

Prior to data analysis, normality tests were employed for all dependent measures. In cases where the distributions were not normal, non-parametric comparisons (independent samples Kruskal-Wallis tests) were utilized; this turned out to be the case for open arm head dips, short-term memory performance in the RAWM and long-term memory performance in the RAWM. However, we also show ANOVA results for these measures for the benefit of the reader. In cases where the distributions were normal, two-way ANOVAs were used to analyze the data; this turned out to be the case for all remaining data. For the two-way ANOVAs, stress (stress, no stress) and drug (metyrapone, vehicle) served as the between-subjects factors. A mixed-model ANOVA was used to analyze arm entry errors on the acquisition trials in the RAWM with stress and drug serving as the between-subjects factors and trial (trials 1-12) serving as the within-subjects factor. Several data from rats trained in the RAWM had to be excluded from the analyses, due to experimenter error. Outlier data points 
that were more than 3 standard deviations beyond the exclusive mean were removed from all analyses. Alpha was set at 0.05 for all analyses.

\section{Results}

\section{Physiological effects}

Corticosterone levels: There were significant main effects of stress, $F(1,34)=15.73$, and drug, $F(1,34)=6.08$, and a significant Stress x Drug interaction, $F(1,34)=19.20\left(p^{\prime} \mathrm{s}<0.05\right)$. Stressed rats treated with vehicle exhibited significantly greater corticosterone levels than all other groups, indicating that metyrapone prevented the stress-induced increase in circulating corticosterone (see Figure 1).

Body weight: For body weight gained following stress exposure, there was a significant main effect of drug, $\mathrm{F}(1,39)=$ $7.24, \mathrm{p}<0.05$, indicating that metyrapone-treated animals gained significantly less weight than vehicle-treated animals. However, there was no significant main effect of stress, $\mathrm{F}(1,39)=2.16$, and the Stress $\mathrm{x}$ Drug interaction was not significant, $F(1,39)=0.70$ (p's > 0.05).

\section{Elevated plus maze}

Open arm behavior: For the amount of time spent in the open arms (see Figure 2A) and the number of entries made into the open arms (see Figure 2B), there were no significant main effects of stress (time: $F(1,39)=0.91$; entries: $F(1,38)=0.29$ ) or drug (time: $F(1,39)=0.23$; entries: $F(1,38)=0.30$ ), and the Stress $\mathrm{x}$ Drug interactions were not significant (time: $F(1,39)=1.22$; entries: $F(1,38)=2.46$ ) (all $p$ 's $>0.05$ ). There was, however, a significant main effect of stress for open arm head dips, $F(1,38)=$ $6.58, p<0.05$, indicating that the stressed rats made significantly fewer open arm head dips than the non-stressed rats (see Figure 2C). There was no significant main effect of drug on open arm head dips, $F(1,38)=0.28$, and the Stress $\mathrm{x}$ Drug interaction was not significant, $F(1,38)=3.04(p$ 's $>0.05)$. A non-parametric Kruskal-Wallis test confirmed the presence of a significant effect across groups for open arm head dips $(p=0.01)$.

Closed arm behavior: For the amount of time spent in the closed arms (see Figure 2D), there were no significant main effects of stress, $F(1,39)=0.91$, or drug, $F(1,39)=0.23$, and the Stress $x$ Drug interaction was not significant, $F(1,39)=1.22$ ( $p$ 's $>0.05$ ). There was, however, a significant main effect of stress for closed arm entries, $F(1,38)=9.02, p<0.05$, indicating that the stressed rats made significantly less closed arm entries than the non-stressed rats (see Figure 2E). There was no significant main effect of drug for closed arm entries, $F(1,38)=1.31$, and the Stress $\mathrm{x}$ Drug interaction was not significant, $F(1,38)=1.00\left(p^{\prime} \mathrm{s}>0.05\right)$.

Rearing: For rearing episodes on the EPM (see Figure 2E), there were no significant main effects of stress, $F(1,39)=0.50$, or drug, $F(1,39)=0.06$, and the Stress $\mathrm{x}$ Drug interaction was not significant, $F(1,39)=0.01$ ( $p$ 's > 0.05).

Overall Movement (Open Arm Entries + Closed Arm Entries): The analysis of overall movement on the EPM revealed a significant main effect of stress, $F(1,38)=4.27, p<0.05$,

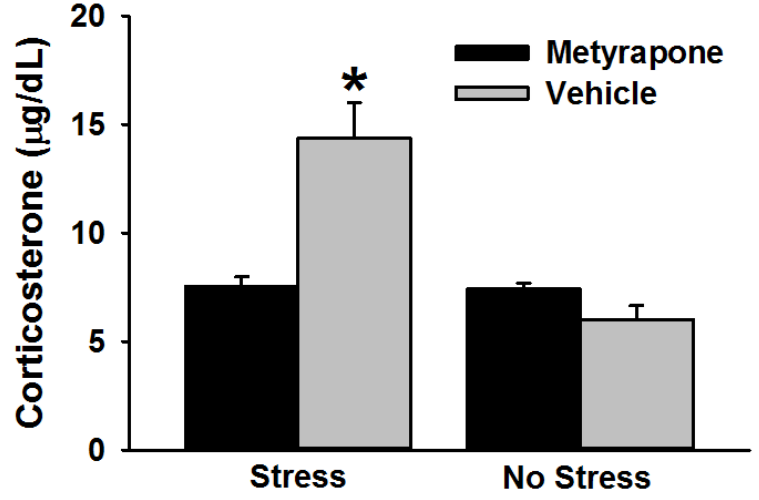

Figure 1: Corticosterone levels in serum samples collected immediately following stress / no stress exposure. Stressed rats treated with vehicle exhibited greater serum corticosterone levels than all other groups; thus, metyrapone effectively blocked the increase in corticosterone that was induced by stress. Data are presented as means \pm SEM. * $p<0.01$ relative to all other groups.

indicating that stressed rats made fewer arm entries, overall, than non-stressed rats. There was no significant main effect of drug, $F(1,38)=0.96$, and the Stress $\mathrm{x}$ Drug interaction was not significant, $F(1,38)=2.14\left(p^{\prime} \mathrm{s}>0.05\right)$.

\section{Radial-arm water maze}

For the acquisition trials (see Figure $3 \mathrm{~A}$ ), there were significant main effects of trial, $F(11,330)=17.08$, and drug, $F(1,30)=7.61$, indicating that the rats made significantly fewer errors as the trials progressed and that rats treated with metyrapone made significantly fewer errors during acquisition than rats treated with vehicle $(p$ 's $<0.01)$. There was no significant main effect of stress, $F(1,30)=0.01$, and the Stress $\mathrm{x}$ Trial, $F(11,330)=1.27$, Stress $x$ Drug, $F(1,30)=1.21$, Drug $\times$ Trial, $F(11,330)=0.56$, and Stress $\mathrm{x}$ Drug $\mathrm{x}$ Trial, $F(11,330)=1.53$, interactions were not significant (all $p$ 's $>0.05$ ).

For the short-term memory trial (see Figure $3 \mathrm{~A}$ ), there was no significant main effect of stress, $F(1,29)=0.08$, or drug, $F(1,29)$ $=0.51$, and the Stress $\mathrm{x}$ Drug interaction was not significant, $F(1,29)=0.70$ ( $p$ 's $>0.05)$. The lack of statistical significance for short-term memory was confirmed by a non-parametric KruskalWallis test $(p=0.81)$.

For the long-term memory trial (see Figure $3 \mathrm{~B}$ ), the main effects of stress, $F(1,29)=3.22$, and drug, $F(1,29)=3.87$, as well as the Stress $x$ Drug interaction, $F(1,29)=3.28$, were all approaching significance $(p$ 's $<0.08)$. A non-parametric Kruskal-Wallis test indicated the presence of a significant effect across groups for long-term memory performance $(p=0.04)$. Examination of the data suggested that these effects were being driven by the stressed rats injected with metyrapone committing more errors, an a priori hypothesis described above. Therefore, we conducted planned comparisons (corrected for multiple comparisons) between the stress-metyrapone group and all other groups on the long-term memory test trial. These comparisons indicated 

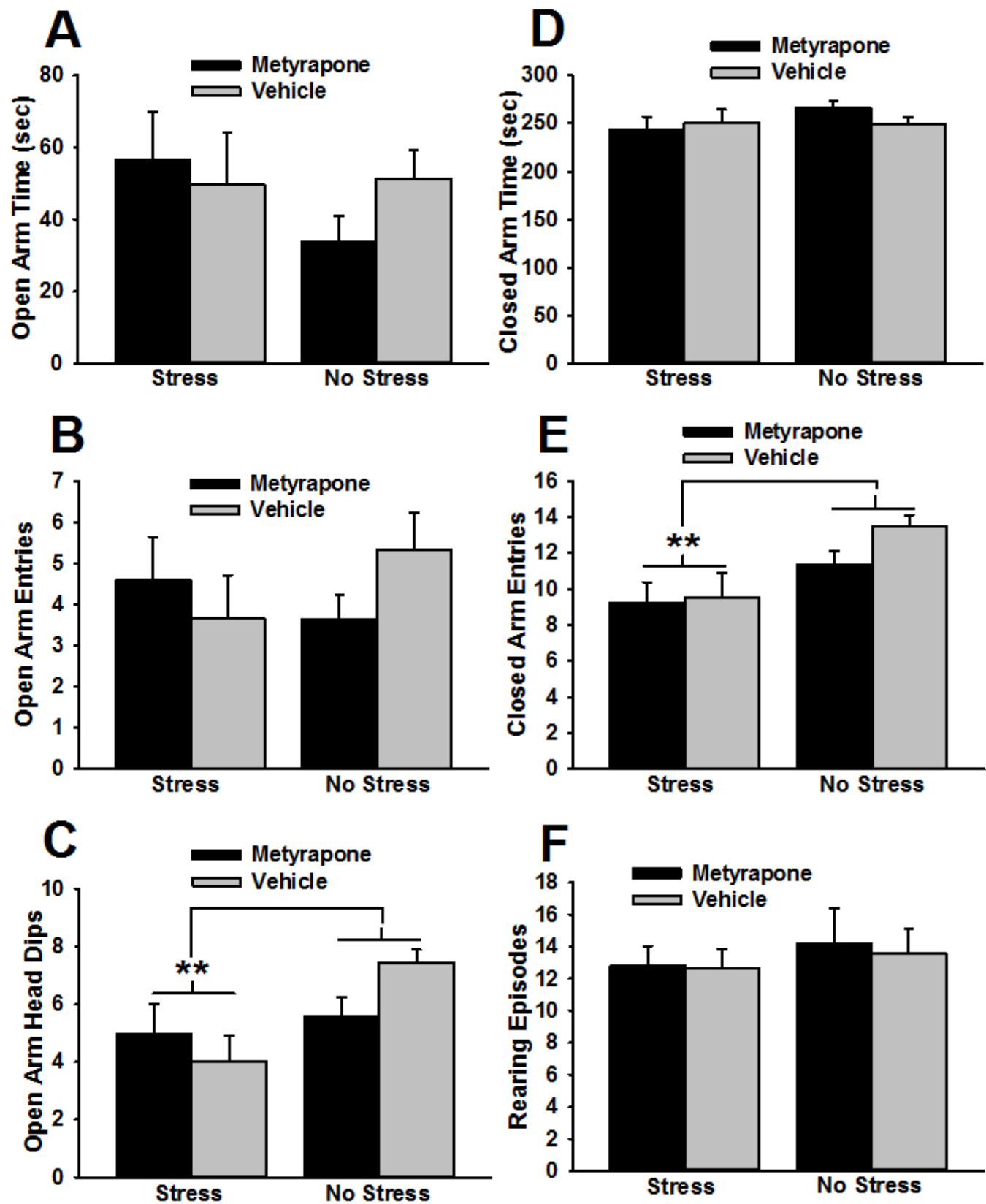

Figure 2: The amount of time spent in the open arms (A), the number of open arm entries committed (B), the number of open arm head dips committed (C), the amount of time spent in the closed arms (D), the number of closed arm entries committed (E) and the number of rearing episodes committed (F) on the elevated plus maze one week following stress exposure. Stressed rats exhibited significantly fewer open arm head dips and closed arm entries than non-stressed rats. Data are presented as means \pm SEM. ${ }^{* *} \mathrm{p}<0.05$ significant main effect relative to no stress.

that the stressed rats treated with metyrapone made significantly more errors on the 24-hr memory test trial than all other groups (p's < 0.05).

\section{Correlational analyses}

We conducted exploratory bivariate correlations between rats' corticosterone levels following stress and the behavioral measures collected one week later. When conducting such analyses for the entire sample, we observed negative relationships between corticosterone levels and open arm entries, $r(37)=$
-0.34 , closed arm entries, $r(37)=-0.35$, and open arm head dips, $r(37)=-0.48(p$ 's $<0.05)$. When these analyses were restricted solely to stressed animals, we observed negative relationships between corticosterone levels and open arm time, $r(18)=-0.52$, open arm entries, $r(18)=-0.58$, and arm entry errors committed on the long-term memory trial, $r(16)=-0.57$ (see Figure 4) ( $p$ 's $<0.05$ ).

\section{Discussion}

Clinical work in humans has suggested that an individual's 
acute physiological response to trauma may affect his or her likelihood of developing PTSD. One such finding has indicated that people who exhibit abnormally low cortisol responses to trauma are more likely to develop PTSD [8-10]. Since experimental work on this topic in humans is practically and ethically impossible, we conducted the present study to examine the effects of a blunted HPA axis response to stress on the development of PTSD-like behaviors in rats. As illustrated in Figure 2, we found that stress exposure, alone, led to less movement and fewer open arm head dips on the EPM, but that the expression of these behaviors was not exacerbated by reduced corticosterone levels during stress exposure. On the other hand, we found that a blunted HPA axis response to stress led to impaired long-term spatial memory in the RAWM (see Figure 3). Although these data are preliminary, they could suggest that reduced corticosterone levels at the time
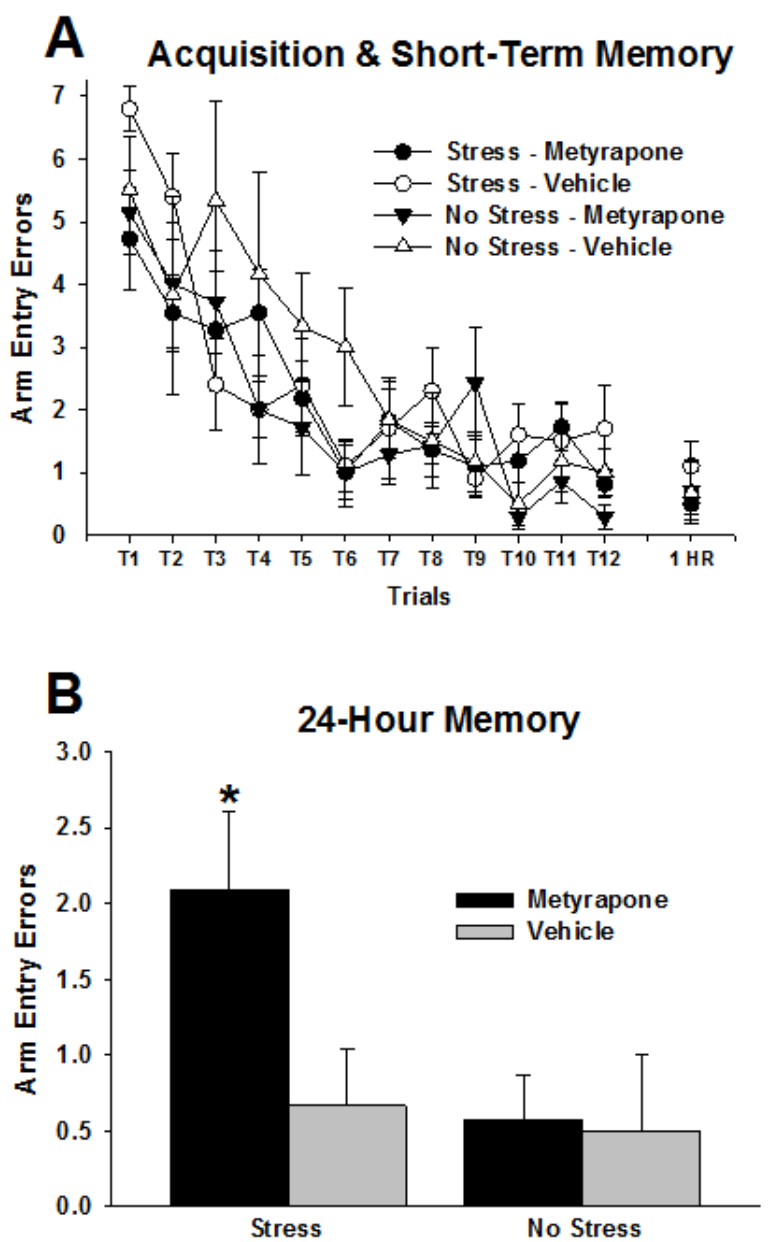

Figure 3: Arm entry errors committed during acquisition / short-term memory testing (A) and long-term memory testing (B) in the RAWM. Acquisition and short-term memory testing took place $24 \mathrm{hr}$ following EPM testing, or 8 days following stress exposure. Long-term RAWM memory testing occurred $24 \mathrm{hr}$ after training, or 9 days following stress exposure. Stressed rats treated with metyrapone committed significantly more arm entry errors on the long-term memory test trial than all other groups. Data are presented as means \pm SEM. $* p<0.05$ relative to all other groups.

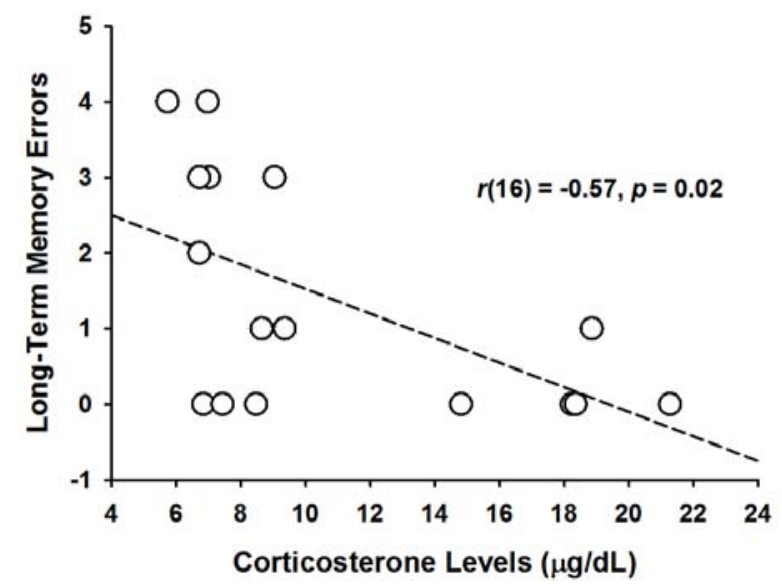

Figure 4: Relationship between corticosterone levels immediately following stress exposure and long-term water maze memory 9 days later in stress-exposed rats. The significant negative correlation suggests that lower corticosterone levels following stress exposure were associated with poorer long-term spatial memory.

of stress result in long-term cognitive impairments and that such an abnormal physiological response to trauma intensifies some, but not all, PTSD-like symptoms in rats.

We did not observe significant effects of stress on body weight gained or several behavioral measures on the EPM, including the most recognized measure of anxiety-like behavior, open arm time (see Figure 2A). We likely did not observe any differences in body weight because we employed a single stress exposure only. Chronic stress studies are more likely to reveal body weight changes in rodents and typically involve several weeks of stress. Nevertheless, we did find that even though the stressed rats spent a similar amount of time on the open arms as the non-stressed rats, they committed fewer open arm head dips (see Figure 2C), indicating a reduction of risk assessment behavior, potentially reflective of increased anxiety. Moreover, the stressed rats moved less in the closed arms (see Figure 2E), which is suggestive of a reduction of overall movement. This was corroborated by the detection of a significant main effect of stress in a subsequent analysis on overall movement (open + closed arm entries). We did not find an exacerbation of such effects as a result of a blunted HPA axis response to stress. If anything, exploratory correlational analyses revealed a negative relationship between corticosterone levels following stress exposure and open arms time, open arm entries, closed arm entries and open arm head dips, suggesting that rats exhibiting greater corticosterone levels following stress exhibited greater anxiety-like behavior on the EPM. This finding suggests that a blunted corticosterone response to stress could actually prevent anxiety-like behavior on the EPM, which is contrary to our hypotheses and contradicts the findings of Bui and colleagues [12]. Our findings may differ from those of Bui and colleagues [12] for a couple of reasons. First, we employed a higher dose of metyrapone in the present study $(150 \mathrm{mg} / \mathrm{kg})$ than that which 
was employed by Bui and colleagues (75 mg/kg). Second, we tested the rats' behavior one week, as opposed to three weeks, following stress exposure. These methodological differences could explain the apparent discrepancy in findings and may also explain why we did not observe general stress effects on all measures of EPM behavior. That corticosterone levels following stress were positively associated with anxiety-like behaviors on the EPM could suggest that only certain PTSD-like symptoms in rats are exacerbated by a blunted corticosterone response to stress. Such a conclusion is consistent with previous work in rodents showing that pharmacologically blocking glucocorticoid receptors after stress exposure can prevent the development of anxiety-like behaviors on the EPM [24].

The most interesting, and novel, finding from the present study was that a blunted HPA axis response to stress resulted in a significant impairment of long-term spatial memory more than a week after stress exposure (see Figure 3B). Exploratory correlational analyses revealed a significant negative correlation between the post-stress corticosterone levels of stressed animals and the number of arm entry errors that they committed in the RAWM on the long-term memory test trial (see Figure 4). These findings cannot be attributed to a general impairment of learning as a result of the blunted HPA axis response to stress because the stressed rats treated with metyrapone exhibited performance during acquisition and the short-term memory trials that was statistically equivalent to all other groups. It is not exactly clear why a blunted HPA axis response to stress would exacerbate its effects on cognitive performance. Clinical research has shown that the administration of glucocorticoids can enhance learning and memory in people with PTSD, while impairing those processes in healthy controls [31-32]. In addition, several studies have reported that the administration of glucocorticoids either shortly after the trauma or after the development of PTSD can prevent / ameliorate the onset / maintenance of PTSD symptoms [33-37]. These findings suggest that the reduced HPA axis activity observed in people who are exposed to trauma or in those individuals who have PTSD could be directly related to PTSD symptom intensity. Thus, although the neurobiological mechanisms underlying the effects observed in the present study are not presently known, they do suggest that a reduced corticosteroid response to stress may intensify subsequent development of PTSD-like cognitive impairments.

The data presented in this manuscript should be interpreted cautiously. Metyrapone is a corticosteroid synthesis inhibitor, meaning that, in addition to reducing corticosterone levels, it likely led to an increase in the levels of corticotropin-releasing hormone and adrenocorticotropic hormone by effectively removing the negative feedback that corticosterone exerts on glucocorticoid receptors. Therefore, it is possible that the presumed elevations of these hormones, which have been implicated in anxiety-like behavior themselves (38), could have influenced the observed effects. Future work will need to assess the levels of these hormones following a manipulation similar to the one reported here and/or examine the influence of such substances on exacerbating PTSD-like behavior development following stress. In addition, determining baseline corticosterone levels before and/or after the stress / no stress manipulation would provide for a more comprehensive characterization of the role of the HPA axis in the onset of PTSD-like behaviors. This is important because even after development of the disorder, people with PTSD typically exhibit abnormally low baseline levels of cortisol, a finding that has been speculated to result from enhanced negative feedback of the HPA axis [39-41]. Thus, it is not known whether abnormally low cortisol levels are a predisposing, baseline characteristic that enhances these individuals' likelihood of developing PTSD following trauma or if such levels develop as a result of the disorder. Finally, in the present study, we studied male rats only. Although this has been a common bias in preclinical models of PTSD, future work needs to examine how female rats are influenced by similar manipulations, especially since extensive work in humans suggests that female are significantly more likely to develop PTSD than males [42].

\section{Acknowledgements}

The authors would like to thank Rebecca Brooks and Robert Carrothers for their significant contribution to this study.

\section{References}

1. Ehlers A, Hackmann A, Michael T (2004) Intrusive re-experiencing in post-traumatic stress disorder: phenomenology, theory, and therapy. Memory 12(4): 403-415.

2. Elzinga BM, Bremner JD (2002) Are the neural substrates of memory the final common pathway in posttraumatic stress disorder (PTSD)? J Affect Disord 70(1): 1-17.

3. Nemeroff CB, Bremner JD, Foa EB, Mayberg HS, North CS, et al. (2006) Posttraumatic stress disorder: a state-of-the-science review. J Psychiatr Res 40(1):1-21.

4. Reynolds M, Brewin CR (1999) Intrusive memories in depression and posttraumatic stress disorder. Behav Res Ther 37(3): 201-215.

5. Speckens AE, Ehlers A, Hackmann A, Ruths FA, Clark DM, (2007) Intrusive memories and rumination in patients with post-traumatic stress disorder: a phenomenological comparison. Memory 15(3): 249 257.

6. Stam R, (2007) PTSD and stress sensitisation: a tale of brain and body Part 1: human studies. Neurosci Biobehav Rev 31(4): 530-557.

7. Ozer EJ, Weiss DS (2004) Who develops posttraumatic stress disorder? Current Directions in Psychological Science 13(4) : 169-172.

8. Delahanty DL, Raimonde AJ, Spoonster E (2000) Initial posttraumatic urinary cortisol levels predict subsequent PTSD symptoms in motor vehicle accident victims. Biol Psychiatry 48(9): 940-947.

9. Ehring T, Ehlers A, Cleare AJ, Glucksman E (2008) Do acute psychological and psychobiological responses to trauma predict subsequent symptom severities of PTSD and depression? Psychiatry Res 161(1) : 67-75.

10. McFarlane AC, Barton CA, Yehuda R, Wittert G( 2011) Cortisol response to acute trauma and risk of posttraumatic stress disorder. Psychoneuroendocrinology 36(5), 720-727.

11. Cohen H, Zohar J , Gidron Y, Matar MA, Belkind D, Loewenthal U, et al. (2006) Blunted HPA axis response to stress influences susceptibility 
to posttraumatic stress response in rats. Biol Psychiatry 59(12) : $1208-1218$

12. Bui VH, Zoladz PR, Conrad CD, Diamond DM (2006) Reduced adrenal and parasympathetic inhibition during traumatic stress: A physiologically relevant animal model of PTSD. Thirty-Sixth Annual Meeting of the Society for Neuroscience. Atlanta, GA.

13. Brewin CR, Smart L (2005) Working memory capacity and suppression of intrusive thoughts. J Behav Ther Exp Psychiatry 36(1): 61-68.

14. Chemtob CM, Roitblat HL, Hamada RS, Muraoka MY, Carlson JG, et al (1999) Compelled attention: the effects of viewing trauma-related stimuli on concurrent task performance in posttraumatic stress disorder. J Trauma Stress 12(2): 309-326.

15. McNally RJ (2005) Debunking myths about trauma and memory. Can J Psychiatry 50(13): 817-822.

16. Moradi AR, Doost HT, Taghavi MR, Yule W, Dalgleish T(1999) Everyday memory deficits in children and adolescents with PTSD: performance on the Rivermead Behavioural Memory Test. J Child Psychol Psychiatry 40(3) :357-361.

17.Zoladz PR, Woodson JC, Haynes VF, Diamond DM(2010) Activation of a remote (1-year old) emotional memory interferes with the retrieval of a newly formed hippocampus-dependent memory in rats. Stress $13(1): 36-52$

18. Jenkins JS, Meakin JW, Nelson DH, Thorn GW, (1958) Inhibition of adrenal steroid 11-oxygenation in the dog. Science 128: 478-479.

19. Burke H M, Robinson C M, Wentz B, McKay J, Dexter KW, et al (2013). Sex-specific impairment of spatial memory in rats following a reminder of predator stress. Stress 16(4): 469-476.

20. Diamond DM, Campbell AM, Park CR, Woodson JC, Conrad CD, et al. (2006) Influence of predator stress on the consolidation versus retrieval of long-term spatial memory and hippocampal spinogenesis. Hippocampus 16(7) : 571-576

21. Roth TL, Zoladz PR, Sweatt JD, Diamond DM, (2011)Epigenetic modification of hippocampal Bdnf DNA in adult rats in an animal model of post-traumatic stress disorder. J Psychiatr Res 45(7): 919926.

22.Zoladz PR, Conrad CD, Fleshner M, Diamond DM, (2008) Acute episodes of predator exposure in conjunction with chronic social instability as an animal model of post-traumatic stress disorder. Stress 11(4): 259-281.

23. Korte SM, De Boer SF (2003) A robust animal model of state anxiety: fear-potentiated behaviour in the elevated plus-maze. Eur J Pharmacol 463(1-3) : 163-175

24. Adamec R, Muir C, Grimes M, Pearcey K (2007) Involvement of noradrenergic and corticoid receptors in the consolidation of the lasting anxiogenic effects of predator stress. Behav Brain Res 179(2): 192-207

25. Cohen H, Kaplan Z, Matar M A, Loewenthal U, Kozlovsky N, et al (2006). Anisomycin, a protein synthesis inhibitor, disrupts traumatic memory consolidation and attenuates posttraumatic stress response in rats. Biol Psychiatry 60(7): 767-776.

26. Sandi C, Woodson JC, Haynes VF, Park CR, Touyarot K, et al. (2005) Acute stress-induced impairment of spatial memory is associated with decreased expression of neural cell adhesion molecule in the hippocampus and prefrontal cortex. Biol Psychiatry 57(8) : 856-864.

27.Zoladz PR, Campbell AM, Park CR, Schaefer D, Danysz W, et al. (2006) Enhancement of long-term spatial memory in adult rats by the noncompetitive NMDA receptor antagonists, memantine and neramexane. Pharmacol Biochem Behav 85(2) : 298-306.

28. Zoladz PR, Woodson JC, Haynes VF, \& Diamond DM (2010) Activation of a remote (1-year old) emotional memory interferes with the retrieval of a newly formed hippocampus-dependent memory in rats. Stress 13(1): 36-52.

29. Williamson DG, O'Donnell VJ(1969) The interaction of metopirone with adrenal mitochondrial cytochrome P-450. A mechanism for the inhibition of adrenal steroid 11 beta-hydroxylation. Biochemistry 8(4): 1306-1311.

30. Park CR, Zoladz PR, Conrad CD, Fleshner M, Diamond DM(2008) Acute predator stress impairs the consolidation and retrieval of hippocampus-dependent memory in male and female rats. Learn Mem 15(4) : 271-280.

31. Wingenfeld K, Driessen M, Terfehr K, Schlosser N, Fernando SC, et al. (2012) in press. Cortisol has enhancing, rather than impairing effects on memory retrieval in PTSD. Psychoneuroendocrinology 37(7): 1048-1056.

32. Yehuda R, Harvey P D, Buchsbaum M, Tischler L, Schmeidler J (2007) Enhanced effects of cortisol administration on episodic and working memory in aging veterans with PTSD. Neuropsychopharmacology 32(12): 2581-2591.

33. Aerni A, Traber R, Hock C, Roozendaal B, Schelling G, et al. (2004) Low-dose cortisol for symptoms of posttraumatic stress disorder. Am J Psychiatry 161(8): 1488-1490.

34. Hauer D, Weis F, Krauseneck T, Vogeser M, Schelling G, et al. (2009) Traumatic memories, post-traumatic stress disorder and serum cortisol levels in long-term survivors of the acute respiratory distress syndrome. Brain Res 1293: 114-120.

35. Schelling G (2002). Effects of stress hormones on traumatic memory formation and the development of posttraumatic stress disorder in critically ill patients. Neurobiol Learn Mem 78(3) : 596-609.

36. Schelling G, Kilger E, Roozendaal B, de Quervain DJ, Briegel J, Dagge A, et al. (2004) Stress doses of hydrocortisone, traumatic memories, and symptoms of posttraumatic stress disorder in patients after cardiac surgery: a randomized study. Biol Psychiatry 55(6) : 627-633.

37. Schelling G, Roozendaal B, Krauseneck T, Schmoelz M,DE Quervain D, et al. (2006) Efficacy of hydrocortisone in preventing posttraumatic stress disorder following critical illness and major surgery. Ann N Y Acad Sci 1071: 46-53.

38. Holsboer F, Ising M (2008) Central CRH system in depression and anxiety--evidence from clinical studies with CRH1 receptor antagonists. Eur J Pharmacol 583(2-3) : 350-357.

39. de Kloet CS, Vermetten E, Geuze E, Kavelaars A, Heijnen CJ, et al. (2006) Assessment of HPA-axis function in posttraumatic stress disorder: pharmacological and non-pharmacological challenge tests, a review. J Psychiatr Res 40(6) : 550-567.

40. Yehuda R (2005) Neuroendocrine aspects of PTSD. Handb Exp Pharmacol (169) : 371-403.

41. Yehuda R (2009) Stress hormones and PTSD. In PJ Shiromani, TM Keane \& JE LeDoux (Eds.), Post-traumatic stress disorder: Basic science and clinical practice. Humana Press, New York, pp. 257-275.

42. Tolin DF, \& Foa EB (2006) Sex differences in trauma and posttraumatic stress disorder: a quantitative review of 25 years of research. Psychol Bull, 132(6), 959-992. 